\title{
Le français chez les étudiants indonésiens en France : leur représentation de la langue, de la formation initiale, et de la pratique linguistique
}

\author{
Neli PURWANI \\ Département de français, Universitas Negeri Semarang - Indonésie \\ Reçu le 20 avril 2018 | Évalué le 25 avril 2018 | Accepté le 29 juin 2018
}

\begin{abstract}
RÉSUMÉ. Cette recherche présente une étude des représentations de 20 étudiants indonésiens de Master 2 et de doctorat, qui sont en programme de collaboration entre une indonésienne et une université française. Nous nous sommes intéressés à leurs représentations de la pratique de la langue française et leur perception des interactions avec des locuteurs natifs et dans l'environnement exolingue. Les données sont collectées de l'enquête distribuée par courriel. L'analyse des données est basée sur les théories de représentation sociale des langues et de l'apprentissage. Le résultat montre que les compétences et les connaissances linguistiques du public de cette recherche se développent grâce à l'interaction exolingue. Pour ce public, il est nécessaire de rajouter le nombre d'heures pour l'apprentissage et la pratique linguistique dans la formation initiale de FLE car la classe de FLE est l'endroit principal où ils communiquent avec leur enseignant qui est, pour eux, un interlocuteur compétent de langue cible.
\end{abstract}

Mots-clés : connaissance et compétence linguistique, interaction, langue, représentations, se développer.

\begin{abstract}
This study discusses the use of French language among 20 Indonesian master students and PhD students who join the collaboration program between a university in Indonesia and in France. The study aims to describe their representations in using French language, and their perception on interacting with native speakers and with "exoling" environment as well. The data is collected by distributing questionnaire via email. The data analysis is formulated using the theories of social representation of languages and learning. The result shows that the students' language skills and knowledge develop through the "exoling" interaction. For this students, it is necessary to add the number of hours of French class (FLE training) and to increase the frequency of linguistic practice during the class, since it appears to be the main place where they communicate with their teacher, who is, for them, a competent interlocutor of the target language.
\end{abstract}

Keywords: interaction, language, linguistics knowledge's and skills, representations, develop.

自 auteur correspondant : npurwani@mail.unnes.ac.id

Pour citer cet article (Style APA) : Purwani, N. (2018). Le français chez les étudiants indonésiens en France : leur représentation de la langue, de la formation initiale, et de la pratique linguistique. Francisola: Revue Indonésienne de la langue et la littérature françaises, 3(1), 45-53. doi: 10.17509/francisola.v3i1.11890 


\section{INTRODUCTION}

Communiquer dans une langue étrangère avec ses locuteurs natifs (LN), pour un locuteur non natif (LNN), est une occasion de découvrir les nuances de discours qui ne pourraient pas être retrouvées dans un manuel scolaire, des gestuelles utilisées ou encore le rythme naturel de cette langue. Un étudiant indonésien faisant ses études en France a l'occasion de consolider ses compétences d'usage de langue. Il doit pratiquer des interactions en français étant donné qu'il est dans le pays de cette langue, en sachant qu'avant son arrivée en France, il a fait une formation initiale de français langue étrangère en Indonésie, il a donc pratiqué le français seulement dans le cadre de la formation linguistique.

Étant en France, il est obligé de parler français aux gens autour de lui. Il vit dans la communauté française pendant des mois, ce qui devient pour lui des moments riches en apprentissage. Associer à sa présence en France, il a des sentiments très variés tels que le doute sur ces capacités de communication, entre autres.

Pour l'étudiant étranger, en l'occurrence l'étudiant indonésien en France, vivre parmi les LNs lui donne beaucoup d'expériences de la pratique linguistique, il peut donc faire sa propre impression sur ses pratiques et sur l'usage de la langue en s'exerçant avec les LN. L'impression, dans ce contexte, est la perception de ses compétences de la pratique de la langue française : sa compétence à comprendre et à parler français lors d'une communication, mais aussi sa perception de la langue française parlée par ses interlocuteurs natifs.

Les expériences de l'utilisation de la langue française chez les étudiants indonésiens en France nous semblent un sujet intéressant à étudier, plus précisément le sujet portera sur les étudiants boursiers de DDIP (Double Degre Indonesia - Perancis), dont la formation initiale de la langue française est organisée à des heures de disponibilités des étudiants voulant suivre cette formation. Ces étudiants boursiers de DDIP sont des étudiants participant au programme de coopération entre deux universités, une université indonésienne et une université française. Ce programme permet aux étudiants de Master ou de doctorats de faire leur première année d'études dans une université en Indonésie, et de terminer leur Master ou doctorat dans une université en France.

Le concept de la pratique de recherche sur la représentation est initié par Jodelet qui a présenté le concept de la représentation sociale. Les études de représentations sociales, selon Jodelet (cité dans Moussouri, 2010, p. 510), “...comportent des éléments informatifs, cognitifs, idéologiques, normatifs, des croyances, valeurs, attitudes, opinions, images etc." Cette étude de représentation sociale s'effectue progressivement dans le domaine de DFLES (Didactique de Français Langue Etrangère et Seconde) (Spaëth, 2016).

S'interroger sur les perceptions des expériences langagières des étudiants DDIP, nous amène à appréhender les représentations sociales de la langue française de ces locuteurs étrangers, les représentations de l'apprentissage, et les représentations de la pratique quotidienne. Cette étude décrit indirectement des traces de l'acquisition de la langue française de LNN à l'environnement exolingue.

Dans le champ des représentations des langues, les études sont développées par les spécialistes de l'apprentissage pour connaître les attitudes et les représentations des sujets vis-à-vis des langues maternelles ou nonmaternel, de leurs usages, de leurs pratiques, et de leurs apprentissages. Par l'étude de la représentation de la langue, les images des langues et les images des locuteurs peuvent être découverts, et dans l'étude des représentations il y a la réflexion des normes, des croyances et attitudes, les compréhensions de position de sujet (Bautier, 1995, p.210). Chaque individu possède sa représentation de sa/ ses langue(s) car il a sa façon à construire son bagage des connaissances.

Calvet (1999, p.158) détaille la notion de la représentation de la langue, "la représentation est la façon dont les locuteurs pensent les pratiques, comment ils se situent par rapport aux autres locuteurs, et comment 
ils situent leurs langues par rapport aux autres langues." Calvet souligne que ces représentations déterminent : 1) des jugements sur les langues et la façon de les parler, 2) des attitudes face aux langues, aux accents, 3) des conduits linguistiques. Donc, fondamentalement, les études des représentations des langues étudient ce que les locuteurs disent et pensent des langues qu'ils parlent et de celles que parlent les autres.

Des recherches sur la représentation de la langue française et de son apprentissage sont faites dans le contexte de FLE et aussi FLS: Spaëth (2016), Julien-Kamal (2013), Moussouri (2010), Fleuret et Armand (2012), Herrera (2011), et Bachir (2012). Julien-Kamal (2013) a fait une recherche sur les représentations linguistiques des étudiants de l'université française en Egypte. Pour cette recherche, l'auteur a utilisé la notion de représentation linguistique qui a un caractère dynamique. Ce caractère dynamique de la représentation de la langue est souligné aussi par Fleuret et Armand (2012) dans leur recherche sur la langue par des enfants haïtiens, et par Herrera (2011) dans sa recherche sur la représentation sociale de langue chez les étudiants mexicains. Les états dynamiques de représentation des langues dans le contexte de l'apprentissage, selon Moore (cité dans Fleuret et Armand, 2012) "se modifient en fonction du contexte où ils sont actualisés et en fonction des variables telles que l'âge, le groupe ethnique, la famille..." L'autre recherche, Bachir (2012), dont le sujet est la représentation des langues en contact, est réalisée en se basant sur le concept de Boyer que dans le terrain (dans la pratique linguistique) de contact des langues, des représentations des langues évoluent. Des recherches sur l'interaction entre l'apprenant et LN de langue cible, faites par Nicolaev (2012) et par Robinson, Mackey, Gass, et Schmidt (2014) montrent la contribution de l'interlocuteur natif à l'évolution de la connaissance et compétence de l'apprenant durant l'interaction. Dans le contexte de l'acquisition de langue dans l'environnement de l'apprentissage, Robinson, Mackey, Gass, et Schmidt (2014) ont expliqué que l'apprenant peut faire des réflexions sur ses pratiques linguistiques grâce à sa conscience durant l'interaction.

Dans cette recherche, notre but est de tenter de décrire les représentations de la langue et de la pratique du français par les étudiants indonésiens en France. Plus précisément, nous voulons décrire leurs perceptions de la pratique de la langue française et le rôle des locuteurs natifs à l'évolution de la connaissance et la compétence linguistique de ces étudiants, leurs réflexions sur leurs compétences linguistiques pendant le séjour en France et leurs réflexions de la formation initiale de français langue étrangère (FLE).

\section{MÉTHODE}

Cette étude est menée sur les étudiants boursiers indonésien du programme de double diplôme français-indonésien de niveau master 2 et doctorat. Pour obtenir les données, nous avons demandé aux étudiants concernés de remplir l'enquête distribuée par courriel. Le questionnaire est rédigé en indonésien pour obtenir des réactions plus profondes, surtout sur les questions ouvertes. Ils ont été développés afin d'obtenir des informations appropriées. Il a été envoyé aux enquêtés par le courriel et la messagerie instantanée. L'information de l'adresse de mail des enquêtés est fourni par l'association des étudiants indonésiens en France (Perhimpunan Pelajar Indonesial PPI Perancis).

\section{RÉSULTATS ET DISCUSSION}

L'échantillon est constitué de 20 étudiants : 17 étudiants du niveau de master et 3 étudiants du niveau de doctorat. Ils sont étudiant en 9 institutions différents : Université Paris 7 (1 étudiant), Université de technologie de Compiègne (4 étudiants), École Central Nantes (1 étudiant), Université Lille 1 (1 étudiant), Université de Nantes (2 étudiant), Agro Paris Tech Nancy (1 étudiant), INSA Lyon (2 étudiants), Université Montpellier 2 (2 étudiants), Sup Agro Montpellier (3 étudiants), Université d'Aix-Marseille (3 étudiants).

Avant leur départ en France, ils ont fait leur formation initiale de FLE aux centres des cours de français dans 4 villes différentes 
: Surabaya, Bandung, Bogor, et Semarang. La formation à Surabaya est organisée par l'IFI (Institut français d'Indonésie) Surabaya. La formation à Bandung et Bogor sont organisées par IFI Bandung. La formation à Semarang est organisée par AFS (Alliance Française de Semarang).

Tous les enquêtés sont des locuteurs natifs d'indonésien. L'indonésien est leur langue nationale mais aussi leur L1. Parmi ces 20 enquêtés, il y en a 18 qui parlent la langue régionale, et il y en a 2 qui ne sont pas locuteur de la langue régional. Les 15 enquêtés parlent javanais comme langue régionale, 1 parle minangkabaunais, et 2 parlent sundanais. Ceux qui parlent aussi langue régionale, ils ont deux L1.

La formation initiale de la langue française pour ces étudiants est réalisée durant la première année de la formation de master ou doctorat. Principalement, l'étudiant se concentre sur son apprentissage afin de réussir l'examen de DELF, car pour s'inscrire dans une université en France, il est obligé d'avoir un niveau de DELF B1 ou B2.

Ces 20 enquêtés ont passé l'examen de DELF à la fin de leur formation de FLE. Il y en a 4 ayant réussi à obtenir le niveau B2. 17 enquêtés ont le niveau B1 et 1 enquêté n'a fait que le niveau A2. Il demeure important de rappeler que la raison principale de séjourner en France pour ce public est de faire des études dans des établissements français. Ces établissements utilisent, bien sûr, le français comme le code de communication. Ils utilisent principalement le français dans l'environnement de leur formation. Selon 75 $\%$ des enquêtés, la langue utilisée dans leur formation est le français. Il y a $15 \%$ qui ont répondu que deux langues sont utilisées : le français et 1 'anglais. $10 \%$ ont répondu qu'ils n'utilisent que l'anglais. Pour le dernier, ce sont deux étudiants dans la formation de doctorats. Ils parlent toujours en anglais avec leur directeur et leur équipe du laboratoire.

Nous pouvons dire que pour ces étudiants DDIP, leur compétence communicative en langue anglaise aide à accélérer les communications avec des interlocuteurs de la communauté académique. Ils ne se soucient pas de leur imperfection en français parce que dans la situation académique ils peuvent utiliser l'anglais.

Les autres endroits/ occasions où les enquêtés utilisent la langue française sont dans la cité universitaire où ils habitent, dans les magasins quand ils font des courses, aux bureaux de service public, pendant les soirées et sorties avec des amis, dans les séminaires, pendant les voyages (ex: quand ils font le covoiturage), ou dans le cours de FLE (pour les enquêtés ayant la formation de FLE). Pour ces enquêtés, leurs interlocuteurs natifs principaux sont leurs professeurs/ directeurs de thèse, les amis de la formation (des cours) à l'université.

Hors de ce cadre, ils parlent aussi français avec les personnels du bureau administratif de l'université, les bibliothécaires, les agents d'entretien, les voisins du lieu d'habitation, le personnel du bureau de services publics où ils s'occupent des documents, et les commerçants. Ces interlocuteurs sont classifiés comme des interlocuteurs secondaires.

Ce public utilise le français comme un code de communication dans des environnements et avec des interlocuteurs variés comme dans le tableau ci-dessus.

Tableau 1. Interlocuteur de communication en français des étudiants et les lieux de l'interaction

\section{Interlocuteurs Lieux de l'interaction}

les professeurs et le directeur de mémoire/ thèse, à la faculté/ au laboratoire/ au lieu de leur formation amis, agent d'entretien, personnel du laboratoire, personnel administratif de la faculté, bibliothécaire.

les voisins

à la cité universitaire (dans la cuisine commune, la salle de travail, terrain des sports)/ l'appartement 
les commerçants

l'agent du bureau d'immigration, l'agent de la

banque
Au marché, au magasin

Aux endroits publics

\subsection{Perception sur les compétences communicatives}

Pour savoir leur perception sur la compétence de communication nous avons demandé aux enquêtés de faire une autoévaluation de leur compétence réceptive et productive.

Leur perception sur leur compétence productive est dans la catégorie suffisante. 50 $\%$ des enquêtés pensent que leur niveau de production est moyen, ils trouvent qu'ils ne parlent ni bien/ ni mal le français. $45 \%$ d'enquêtés pensent qu'ils ont un bon niveau en production mais ils ont besoin de temps pour formuler leurs phrases. $5 \%$ d'enquêtés pensent qu'ils n'ont pas de bon niveau en production.

Leur perception sur leur compétence réceptive est mieux que celle de leur compétence productive. Leur perception sur leur compétence réceptive est dans la catégorie élevée. Ils se sentent à l'aise en compréhension. Le résultat de l'enquête montre que $50 \%$ d'enquêtés pensent qu'ils comprennent bien le français dans la communication avec les LN mais avec un peu de temps pour cerner le message. $5 \%$ des enquêtés pensent qu'ils ont de bonne compétence en compréhension. $40 \%$ pensent que leur compétence de compréhension est "juste" ou moyenne. Pour le reste, $5 \%$ pensent qu'ils n'ont pas assez de compétence pour comprendre les paroles de leurs interlocuteurs natifs. Ceux-ci préfèrent communiquer en anglais.

\subsection{Les problèmes dans les communications avec $\mathrm{LN}$ et les solutions}

Des facteurs linguistiques qui entravent la compréhension des enquêtés dans les communications avec LN sont ; 1) le débit de la parole des LN, 2) les mots utilisés par LN qui sont parfois non-familier pour les enquêtés, 3) la prononciation des LN qu'ils trouvent différente, 4) la structure de phrase qu'ils ne comprennent pas, et 5) l'usage des expressions (idiomatique ou figé) dont ils ne connaissent pas les sens.

La prononciation des LN peut être difficile à comprendre par LNN parce que LNN ne sont pas bien habitué, ils sont plus habitués à celle des enseignants de leur formation initiale. LN parle de façon "standard" avec ses appareils articulatoires pour produire des énoncés en sa langue. Des expressions utilisées par LN peuvent causer un problème de compréhension chez LNN car ce dernier ne connaît pas tous ces expressions.

Ayant des obstacles dans la communication, LNN ont appliqué leurs propres stratégies pour en résoudre. Dans le cas de compréhension, la stratégie la plus utilisée par les enquêtés est demander la répétition/ reformulation. Ils demandent de répéter pour pouvoir mieux comprendre des énoncés qu'ils ne captent pas bien, ou demandent de reformuler en phrase plus simple utilisant d'autres mots. La deuxième stratégie est faire semblant pour conserver leur face en souriant ou riant. La troisième stratégie est chercher le sens au dictionnaire/ Google. Quelques enquêtés ont mentionné 3 autres stratégies : demander des éclaircissements à l'autre participant de la conversation, faire une expression gestuelle expliquant l'incompréhension, et demander une explication en anglais.

Dans le cas de production, les enquêtés font des stratégies pour faire comprendre leurs interlocuteurs en reformulant leurs phrases. La répétition permet à un locuteur de perfectionner la qualité de sa prononciation, le choix des mots, et la construction syntaxique. Ils tentent de faire comprendre leur interlocuteur avec leur compétence limitée en langue française. Cet effort permet à l'interlocuteur natif d'offrir des corrections. L'initiation de LNN de produire un énoncé acceptable peut pousser LN à l'aider. Selon le concept des 
mécanismes de réparations de l'énoncé, c'est un cas de l'hétéro-réparation - auto-initiation, LNN ayant l'initiation essaie à parler et LN l'accomplit.

\subsection{Le rôle de $\mathrm{LN}$ dans l'évolution de la connaissance et de la compétence linguistique}

Dans les études de l'acquisition des langues étrangères, un LN est considéré comme le fournisseur des "inputs" les plus appropriés, un individu compétent qui est capable d'identifier les mal formés des expressions grammaticales dans sa langue et de sélectionner des mots appropriés dans chaque contexte. C'est une des définitions de mots "locuteur-natif". Plusieurs auteurs ont expliqué la notion de terme "locuteur-natif". Nous présentons l'explication de Davies (2013, p.3) sur les caractères des LN qui, selon lui, peuvent être explicité des 3 perspectives linguistiques, sociolinguistiques, et psycholinguistiques. Nous pouvons souligner que 1) un locuteur natif possède sa L1 depuis sa naissance ; 2) il a une connaissance intuitive de la langue; 3 ) il est compétent, et capable de produire couramment, le discours spontané en différentes situations; et 4) au niveau le plus haute, il est capable d'écrire et d'interpréter des textes/ discours en différentes formes.

LN ont une contribution à l'évolution de la connaissance et la compétence linguistique des LNN. Tous les enquêtés de cette recherche ont affirmé que les LN peuvent enrichir leur bagage de connaissance linguistique. $50 \%$ d'enquêtés pensent aussi que cela peut aider à consolider la connaissance linguistique qu'ils ont obtenue avant. 18 de nos 20 enquêtés ont donné leur réponse à la question sur le rôle des interlocuteurs natifs de la langue française à la pratique linguistique des enquêtés. Nous avons catégorisé ces 18 réponses des rôles de LN comme ci-dessous :

1. LN corrige la prononciation.

2. LN aide à choisir un mot/ une expression convenant au contexte.

3. LN enrichie le bagage lexical.

4. LN corrige la structure de phrases.

5. LN corrige le malentendu.
6. De LN, l'enquêté s'habitue à écouter et comprendre la langue français version LN.

7. De LN, l'enquêté apprend la mimique, la gestuelle, et l'intonation.

\subsection{Vision sur l'avantage de la maîtrise de la langue française}

Nous avons demandé aux enquêtés de mentionner leur vision sur l'avantage de la maîtrise de la langue française. Ils ont répondu à cette question que nous pouvons catégoriser comme suit: (1) l'avantage du côté économique, (2) l'avantage du côté pratique, (3) L'avantage du côté personnel (le prestige). La première est la réponse qui a un rapport avec une possibilité de travailler dans une entreprise française. La deuxième réponse est celle qui a un rapport avec le besoin de la communication quotidienne. La dernière est celle qui a un rapport avec la satisfaction personnelle telles que : avoir des amis français, et pouvoir parler une autre langue étrangère.

\subsection{Les avis des enquêtés sur la formation initiale}

Nous avons aussi demandé des perceptions des enquêtés sur la formation initiale. Ils ont donné des avis de l'heure des cours, les cours, et les enseignants. Ces avis sont détaillés dans des paragraphes suivants.

Les avis des enquêtés par rapport à l'heure des cours de la formation sont positif : 8 enquêtés montrent leur satisfaction sur l'horaire de la formation de FLE qu'ils ont assisté. Ils trouvent que la durée de la formation n'était pas longue, la formation s'est faite en mode intensif, l'horaire était bien organisé et efficace. Les restes expriment une insatisfaction par rapport au volume d'heure totale de cours qu'ils ont trouvé insuffisant pour bien apprendre la langue, et aussi que l'horaire de la formation d'études de Master ou Doctorat de 1 ${ }^{\mathrm{er}}$ année et celui de cours de français ne sont pas harmonisés. En plus, souvent le cours de français est organisé à la fin de l'après-midi, après une journée des cours de doctorat ou master.

Les avis des enquêtés par rapport aux cours: $65 \%$ d'enquêtés ont donné des réponses exprimant leur satisfaction. Ils ont 
constaté qu'il n'y avait pas de problème avec les sujets des cours, ils les ont trouvés intéressants. Les sujets donnés dans le cours, selon eux, sont la grammaire de la langue française, la culture française, et aussi le vocabulaire.

$35 \%$ d'enquêtés n'ont pas donné des réponses exprimant leur satisfaction, ils ont montré des points faibles du cours de français, tels que: les enseignants profitent $\mathrm{du}$ temps pour expliquer les sujets de cours afin que les élèves puissent les cerner, et que l'interaction n'est pas assez pratiquée. Autres expressions d'insatisfactions par rapport au cours sont comme suit: 1) le cours est trop concentré sur l'examen de DELF, et 2) l'utilisation du manuel de FLE était très dominante alors qu'il manque l'activité d'écoute des dialogues quotidiens qui contiennent des expressions habituelles et utiles pour l'apprenant.

Les avis des enquêtés par rapport aux enseignants : $65 \%$ des enquêtés montrent la satisfaction quant à la qualité des enseignants et leur enseignement. Les $35 \%$ des enquêtés montrent l'insatisfaction et aussi la satisfaction. L'insatisfaction exprimée concerne la façon moins intéressante de professeur de donner le cours.

Ces 20 enquêtés expliquent leur avis, leur conseille pour le perfectionnement de la formation initiale du FLE. Ces avis sont comme suit: 1). L'augmentation de nombre d'heure pour la pratique productive car la classe est le seul endroit où l'étudiant a l'occasion pour pratiquer en tandem avec l'enseignant qui est pour lui un interlocuteur compétent. Dans ce cas nous pouvons dire que pour l'apprenant $\mathrm{du}$ français en Indonésie, la pratique durant le cours est important. Ils pourraient s'entraîner avec leurs enseignants comme les seuls interlocuteurs appropriés. 2). La réorganisation de l'horaire de formation pour que l'étudiant puisse optimiser son apprentissage. 3). La compréhension et la production orale sont deux types de compétences linguistiques qui devraient être entraînées. 4). La classe devrait être reconditionnée à l'usage de français en invitant l'étudiant à utiliser le français le plus souvent possible. L'usage de l'indonésien est seulement pour facilitér le transfert des connaissances déclaratives. 5). Varier l'application des stratégies d'enseignement est nécessaire pour que le cours soit interactif. Et 6). Faire connaître les apprenants au vocabulaire du domaine de cours de master et aux expressions de la vie quotidienne est aussi nécessaire.

\section{CONCLUSION}

Nos enquêtés ont donné une évaluation de leurs compétences linguistiques en français, ils ont expliqué aussi des contributions de leurs interlocuteurs natifs à l'évolution de leur connaissance et compétence linguistique. Dans la communauté académique (à la faculté/ au laboratoire de recherche), les enquêtés peuvent utilisés le français et l'anglais. Quand ils rencontrent quelques difficultés pour s'exprimer en français ou pour comprendre des mots français, leurs interlocuteurs acceptent l'utilisation de l'anglais. Dans les autres communautés, le français est la seule langue acceptable. Dans la question de l'auto-évaluation, la plupart des enquêtés ont de bonne perception de leur compétence de la compréhension de la langue française. Pour la compétence productive, la moyenne des enquêtés pense qu'ils ne parlent ni mal ni bien le français. En général, ils se sentent à l'aise dans l'interaction avec LN.

Les communications "exolingues" sont identiques avec l'inégalité de la compétence qui provoque l'incompréhension et pour se comprendre les interlocuteurs, LN et LNN, font chacun des efforts. Au côté de LNN les difficultés dans la communication sont causées par des raisons différentes. Dans les statistiques, nous voyons que les trois grands facteurs qui entravent les compréhensions des enquêtés dans leurs communications avec LN sont la vitesse de LN, les mots utilisés par LN, et la prononciation de LN. LN parlent de façon normal: au niveau de la rapidité du débit, du choix des mots, de l'articulation standard. Et ceci est difficile pour LNN parce qu'il ne s'est pas bien habitué avec les sons produits et n'a pas le même niveau de bagage lexical. Au début, LNN qui ne s'est pas encore adapté, a 
l'impression que LN parle de façon très rapide et utilise le lexique que LNN ne connaît pas, il entend une langue qui est totalement différente avec celle de ses enseignants de la formation initiale.

Dans les situations où les enquêtés trouvent des difficultés dans la compréhension, la stratégie de demander de rééxpliquer en français est la stratégie la plus prise par les enquêtés. Ils préfèrent garder le contact avec leurs interlocuteurs natifs. Donc ils demandent la réexplication et cette stratégie signale au LN de modifier son discour en mode "xénolecte" afin qu'à la fin, ils pourraient mieux capter ce que dit LN. Dans les interactions, les enquêtés n'ont pas toujours essayé de comprendre tous les messages de communication, ils font aussi parfois semblant de comprendre, pour continuer de participer à l'interaction mais aussi pour garder la face. Après ces deux stratégies, il y a quatre stratégies appliquées par les enquêtés : ils profitent de la présence de technologie "google traduction", la présence de l'autre participant d'interaction pour avoir des éclaircissements, et leur corps pour faire des gestuelles afin d'exprimer leur incompréhension.

LNN ne fait que des efforts pour pouvoir comprendre les messages de l'interaction de LN, mais au niveau de production, avec sa compétence limitée, il est aussi obligé de faire des efforts pour pouvoir bien transmettre les messages de ses énoncés à ses interlocuteurs natifs. Pour résoudre ce problème, nos enquêtés ont fait des stratégies comme la répétition ou la reformulation, la rééxplication en anglais, l'usage des expressions gestuelles, et la dernière stratégie est l'usage du dictionnaire ou "google traduction" pour trouver les mots qu'ils voulaient dire.

Nos enquêtés trouvent que l'interaction avec des LNs donnent des contributions à l'évolution de leurs connaissance et compétence linguistique. Nos enquêtés ont aussi avoué que leurs interlocuteurs natifs donnaient des corrections et des remarques à leurs phrases, au choix des mots, et à la structure des phrases. Les LN corrigent le mal compris. Les LN sont aussi partenaires du tandem linguistique avec lesquels les LNN puissent s'entraîner à parler en français. Pour les enquêtés, LNN leur fournit des inputs linguistiques : le lexique en forme de mots et d'expression, la connaissance sur la grammaire, la connaissance sur la prononciation, la connaissance pragmatique sur la politesse. Selon certain enquêtés, ces inputs lexicaux sont en forme des mots d'argot, de l'abréviation, des expressions quotidiennes et aussi idiomatiques. L'input au type de grammaire/ syntaxe mentionné par deux de nos enquêtés sont la syntaxe des phrases oraux: l'absence de ne dans la négation et l'usage de pronom possessif.

Pour nos enquêtés, ils pensent qu'il y a l'avantage de la maîtrise de la langue française. La plupart sont fiers de la maîtrise de langue. Ils trouvent que le français est une langue globale après l'anglais, et aussi une langue à l'ONU. En maîtrisant cette langue ils pensent pouvoir accéder au marché de travail des entreprises françaises, et pouvoir survivre en France et avoir des amis francophones.

Pour les étudiants DDIP, la formation initiale de la langue française a été organisée en Indonésie durant $4-5$ mois. Cette formation était l'après-midi ou en fin d'après-midi, comme le matin ils ont du assister la classe de leur formation académique à la faculté. Pour cette raison nos enquêtés trouvent que l'organisation de l'horaire de la formation n'était pas assez acceptable pour soutenir l'attente de la formation, arriver au niveau de DELF B1.

\section{REMERCIEMENTS}

Cette recherche a pu être réalisée grâce à la contribution et au soutien de plusieurs personnes à qui je voudrais exprimer ma gratitude et ma reconnaissance. Je souhaite remercier tout particulièrement, Monsieur Bruno Maurer qui a accepté de diriger ce travail. Je souhaite aussi remercier Yness qui a lu pour donner la correction, et les étudiants du programme DDIP 2014/2015 qui ont contribué par l'enquête que j'avais distribuée. 


\section{RÉFÉRENCES}

Bachir, B. (2012). Plurilinguisme et représentation des langues en contact en Algérie. Synergie Chilie, 8, 8384. Repéré à https://gerflint,fr/Base/ Chili8/bessai_bachir.

Bautier, E. (1995). Pratiques langagières, pratiques sociales: de la sociolinguistique à la sociologie $d u$ langage. Paris: Harmattan.

Calvet, L. et Dumont. P. (1999). L'enquête sociolinguistique. Paris: Harmattan.

Davies, A., (2013). Native speakers and native users. Cambridge: Cambridge University Press.

Fleuret, C., et Armand, F., (2012). Le créole c'est presque la même affaire du français, on fait des formules... c'est déformé : Les représentations des langues par des enfants haïtiens. Revue canadienne de linguistique appliquée, 15(1), 42-59. Repéré à https:/journals.lib.unb.ca/

Herrera, A. V., (2011). Représentations sociales de la langues française et motivations de son apprentissage: enquête d'étudiants universitaires méxicains spécialistes et non spécialistes. Synergie Mexique, 1, 57-69. Repéré à https://gerflint.fr/Base/Mexique1/adeli na_velazquez

Julien-Kamal, C., (2013). Pratique et représentation linguistiques des étudiants de la faculté des langues appliquées de l'université française d'Egypte et de leur famille. Reherche en didactique des langues et des cultures, 10(3), 1-12. doi: 10.4000/rdlc.2562

Moussouri, E. (2010). Pratiques didactiques et représentations: un outil pour la conception d'une formation destinée aux enseignants des langues secondes/ d'origine. Recherche en didactique des langues et des cultures, doi: $10.4000 /$ rdlc. 2153

Nicolaev, V. (2012). L'apprentissage du FLE dans un dispositif vidéoeographique synchrone : étude des séequences métalinguistiques. (Doctorat en science du langage, Ecole Normale Supérieure, Lyon). Repéré à: https://tel.archivesouvertes.fr

Robinson, P., Mackey, A., Gass, S. M., et Schmidt, R. (2014). Attention and awareness in second language acquisition. Dans S. M. Gass and A. Mackey (dir.), The Routledge Handbook of Second Language Acquisition (p.247267). Padstow, Cornwall, GrandeBretagne : TJ International Ltd.

Spaëth, V., (2014). La transposition du concept de représentation en didactique $\mathrm{du}$ français langue étrangère et seconde. Recherches et Applications - FDLM, 55(3). Repéré à https://halshs.archivesouvertes.fr/halshs-01322998 